06

\title{
Структурная трактовка изменения свойств нанокомпозитов полимер/углеродные нанотрубки у порога перколяции нанонаполнителя
}

\author{
(С) Г.В. Козлов, И.В. Долбин ฯ \\ Кабардино-Балкарский государственный университет им. Х.М. Бербекова, \\ 360004 Нальчик, Россия \\ ฯ e-mail: i_dolbin@mail.ru \\ Поступило в Редакцию 20 марта 2019 г. \\ В окончательной редакции 20 марта 2019 г. \\ Принято к публикации 22 апреля 2019 г.
}

\begin{abstract}
Структура нанонаполнителя в полимерной матрице нанокомпозитов полимер/углеродные нанотрубки может быть охарактеризована размерностью каркаса нанонаполнителя, которая служит прямым показателем уровня его агрегации. Процесс формирования такого каркаса рассмотрен как результат взаимодействия матричного полимера и углеродных нанотрубок, что позволяет определить его размерность в рамках фрактального анализа. Обнаружено, что размерность каркаса углеродных нанотрубок изменяется как качественно, так и количественно у их порога перколяции, отражая разный уровень агрегации. Указанная размерность однозначно определяет степень усиления рассматриваемых нанокомпозитов и их структурное состояние как системы.
\end{abstract}

Ключевые слова: нанокомпозит, углеродные нанотрубки, структура, каркас нанонаполнителя, уровень агрегации, степень усиления, фрактальная размерность.

DOI: $10.21883 / J T F .2019 .10 .48176 .101-19$

\section{Введение}

В настоящее время углеродные нанотрубки [1] и графены [2] рассматриваются как наиболее перспективные нанонаполнители для полимерных нанокомпозитов. Этот постулат базируется на исключительно высоких механических свойствах и большой степени анизотропии этих нанонаполнителей [3]. Однако свойства нанокомпозитов, наполненных этими нанонаполнителями, не всегда оправдывают оптимистические ожидания. Это обусловлено хорошо известным фактом: нанокомпозиты представляют собой структурно сложные системы, чьи свойства определяются значительным числом факторов, часто взаимосвязанных между собой - агрегацией (структурой) нанонаполнителя в полимерной матрице, его ориентацией межфазными эффектами и т. п. [4].

Как хорошо известно [5,6], для упомянутых выше анизотропных нанонаполнителей наблюдается общий эффект: при очень малых содержаниях нанонаполнителя реализуется сильное улучшение механических свойств, которое далее сменяется их резким дискретным снижением. Обычно этот эффект связывается с достижением нанонаполнителем своего порога перколяции $\varphi_{c}[6,7]$. Однако количественная структурная модель этого эффекта еще не разработана. С физической точки зрения достижение $\varphi_{c}$ означает переход структуры нанонаполнителя от системы отдельных его агрегатов к их непрерывному каркасу [8]. Кроме того, следует учитывать еще один очень важный структурный аспект, а именно все основные структурные компоненты полимерных нанокомпозитов (полимерная матрица [9], агрегаты на- нонаполнителя [1], каркас частиц нанонаполнителя [10] и т.д.) являются фрактальными объектами, что делает обязательным их описание в рамках фрактального анализа [11]. Поэтому целью настоящей работы является количественное описание в рамках фрактального анализа структурного перехода нанонаполнителя (углеродных нанотрубок) у порога перколяции и моделирование влияния этого перехода на модуль упругости нанокомпозитов.

\section{Методика эксперимента}

В настоящей работе выполнен анализ результатов, полученных авторами работы [5], где в качестве нанонаполнителя были использованы многослойные углеродные нанотрубки (МУНТ), имеющие диаметр $16.6 \pm 3.9 \mathrm{~nm}$ и длину $1.20 \pm 0.6 \mu \mathrm{m}$. Указанные МУНТ функционализировались группами -OH для получения смеси с полиметилметакрилатом (ПММА). Содержание МУНТ в рассматриваемых нанокомпозитах варьировалось в пределах 0.065-1.30 wt.\% [5].

Нанокомпозиты ПММА/УНТ синтезированы методом радикальной полимеризации in situ. Пленки нанокомпозитов толщиной $75 \mu \mathrm{m}$ получены методом полива их растворов в толуоле на поверхность тефлона. Для испытаний использовались образцы в виде полос размером $10 \times 2.5 \mathrm{~mm}[5]$.

Механические испытания на одноосное растяжение пленочных образцов нанокомпозитов ПММА/УНТ выполнены на приборе для растяжения Zwick модели 
Z100 при температуре $293 \mathrm{~K}$ и скорости деформации $\sim 10^{-3} \mathrm{~s}^{-1}[5]$.

\section{Результаты и их обсуждение}

Как хорошо известно $[1,4,12,13]$, углеродные нанотрубки в полимерной матрице нанокомпозитов формируют кольцеобразные структуры (или их фрагменты) радиуса $R_{\mathrm{CNT}}$, которые являются фрактальными объектами. Величину $R_{\mathrm{CNT}}$ можно определить с помощью следующего уравнения [14]:

$$
b_{\alpha}=56\left(R_{\mathrm{CNT}}^{2}-0.022\right) \text {, }
$$

где $b_{a}-$ безразмерный параметр, который является характеристикой уровня межфазной адгезии, а $R_{\mathrm{CNT}}$ определяется в $\mu \mathrm{m}$.

Параметр $b_{\alpha}$ определен с помощью перколяционного соотношения [15]

$$
\frac{E_{n}}{E_{m}}=1+11\left(2.8 b_{\alpha} \varphi_{n}\right)^{1.7}
$$

где $E_{n}$ и $E_{m}-$ модули упругости нанокомпозита и матричного полимера соответственно (отношение $E_{n} / E_{m}$ принято называть степенью усиления нанокомпозита), $\varphi_{n}$ - объемное содержание нанонаполнителя, принятое для рассматриваемых нанокомпозитов согласно данным работы [5].

Формирование каркаса частиц (агрегатов частиц) нанонаполнителя ниже и выше порога перколяции существенно различается. В первом случае указанный каркас формируется как результат взаимодействий агрегатов (кольцеобразных структур углеродных нанотрубок) и полимерной матрицы, а во втором - как результат взаимодействий агрегатов углеродных нанотрубок между собой. Это обстоятельство позволяет определить размерность каркаса частиц нанонаполнителя $D_{N}$ следующим образом [16]:

$$
D_{N}=\frac{d\left(2 D_{1}-D_{2}\right)}{d+2\left(D_{1}-D_{2}\right)}
$$

где $d$ - размерность евклидова пространства, в котором рассматривается фрактал (очевидно, в нашем случае $d=3), D_{1}$ и $D_{2}-$ размерности взаимодействующих при формировании каркаса нанонаполнителя структурных компонент.

Как следует из приведенного выше краткого обсуждения, ниже порога перколяции углеродных нанотрубок $\left(\varphi_{n}<\varphi_{c}\right) D_{1}=d_{f}$ и $D_{2}=D_{f}$, где $d_{f}$ и $D_{f}-$ фрактальные размерности структуры полимерной матрицы и агрегатов (кольцеобразных структур) углеродных нанотрубок соответственно, а выше порога перколяции $\left(\varphi_{n} \geq \varphi_{c}\right) D_{1}=D_{2}=D_{f}$.

Рассмотрим методы расчета размерностей $d_{f}$ и $D_{f}$. Величину $d_{f}$ можно определить, согласно уравнению [9]:

$$
d_{f}=(d-1)\left(1+v_{n}\right),
$$

где $v_{n}$ - коэффициент Пуассона, оцениваемый по результатам механических испытаний с помощью соотно-

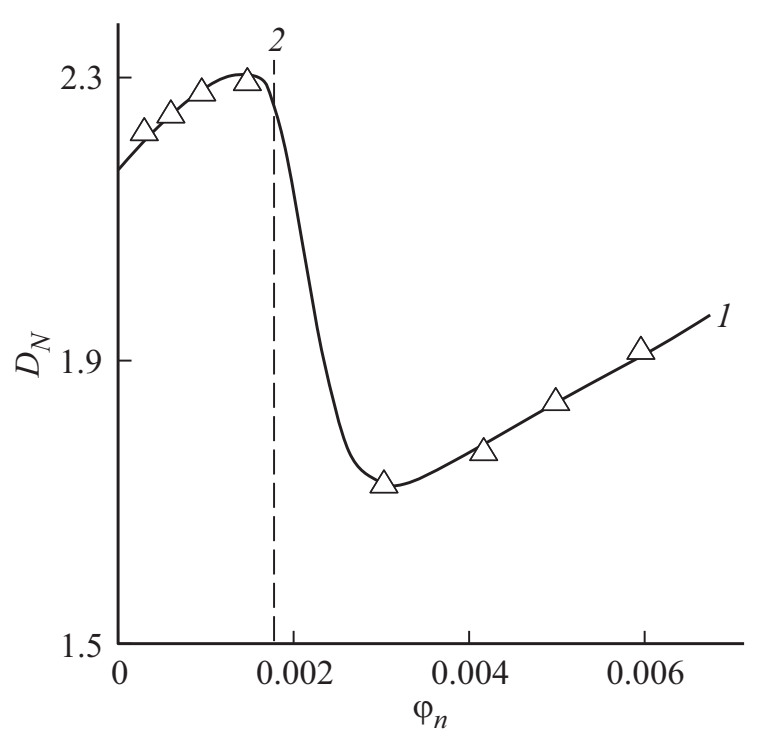

Рис. 1. Зависимость размерности каркаса углеродных нанотрубок $D_{N}$ от объемного содержания нанонаполнителя $\varphi_{n}$ для нанокомпозитов ПММА/МУНТ. Вертикальная штриховая линия 2 указывает порог перколяции углеродных нанотрубок $\varphi_{c}$.

шения [9]:

$$
\frac{\sigma_{Y}}{E}=\frac{1-2 v}{6\left(1+v_{n}\right)}
$$

где $\sigma_{Y}$ и $E-$ предел текучести и модуль упругости полимерного материала.

Размерность $D_{f}$ определена применением следующего уравнения, полученного в рамках модели необратимой агрегации [17]:

$$
R_{\mathrm{CNT}}=3.4 \varphi_{n}^{-1 /\left(d-D_{f}\right)},
$$

где $R_{\mathrm{CNT}}$ задается в $\mathrm{nm}$.

На рис. 1 приведена зависимость размерности каркаса углеродных нанотрубок в полимерной матрице $D_{N}$ от объемного содержания нанонаполнителя $\varphi_{n}$ для нанокомпозитов ПММА/МУНТ. Как можно видеть, при $\varphi_{n}=\varphi_{c}$ происходит резкий спад размерности $D_{N}$, обусловленный изменением взаимодействий углеродные нанотрубки-полимерная матрица на взаимодействия углеродных нанотрубок между собой. Величину порога перколяции $\varphi_{c}$ наиболее просто можно оценить следующим образом [18]:

$$
\varphi_{c}=\frac{\pi}{12}\left(\frac{d_{\mathrm{CNT}}}{2 L_{\mathrm{CNT}}}\right)
$$

где $d_{\mathrm{CNT}}$ и $L_{\mathrm{CNT}}$ - диаметр и длина углеродных нанотрубок, а значение $\varphi_{c}$ при указанных выше величинах $d_{\mathrm{CNT}}$ и $L_{\mathrm{CNT}}$ равно 0.0018 (указано вертикальной штриховой линией 2 на рис. 1$)$.

Авторы $[7,10]$ показали, что в случае перколяционных кластеров их критические индексы для первого, второго и третьего подмножеств связаны с размерностью этих 


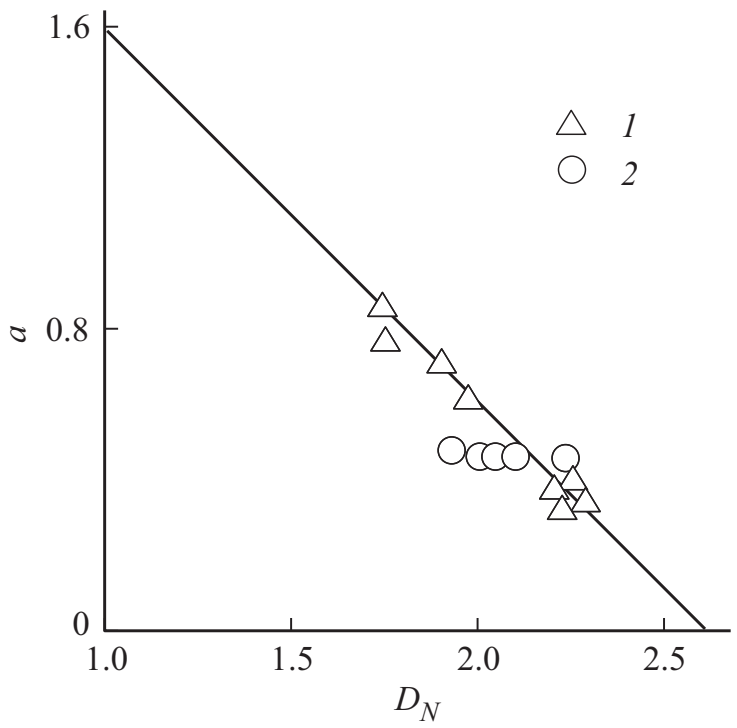

Рис. 2. Зависимость критического индекса $a$ в уравнении (11) от размерности каркаса углеродных нанотрубок $D_{N}$ для нанокомпозитов ПММА/МУНТ (1) и ПА-6/УНТ (2).

кластеров $d_{f}$ следующими соотношениями:

$$
\begin{gathered}
\beta=\frac{1}{d_{f}}, \\
v=\frac{2}{d_{f}}, \\
t=\frac{4}{d_{f}} .
\end{gathered}
$$

Из уравнений (8)-(10) следует, что универсальность критических индексов перколяционной системы связана с ее фрактальной размерностью [7]. В работе [7] были конкретизированы указанные выше подмножества применительно к структуре полимерных нанокомпозитов следующим образом: первым подмножеством $(n=1)$ являются межфазные области, вторым $(n=2)-$ совокупность межфазных областей и нанонаполнителя и третьим $(n=4)$ - собственно наполнитель. В соответствии с этой градацией можно рассматривать истинные, промежуточные нанокомпозиты и микрокомпозиты соответственно [19].

Общее перколяционное соотношение для определения степени усиления $E_{n} / E_{m}$ нанокомпозитов имеет следующий вид [7]:

$$
\frac{E_{n}}{E_{m}}=1+11\left(\varphi_{n}\right)^{a},
$$

где перколяционный индекс $a$ близок (не обязательно равен) к критическим индексам перколяционной системы $\beta, v$ и $t$ в зависимости от армирующей компоненты нанокомпозитов. В рамках такой трактовки $a=1 / d_{f}$ при $\varphi_{n}<\varphi_{c}$ и $a=2 / d_{f}$ при $\varphi_{n} \geq \varphi_{c}[7]$.

На рис. 2 приведена зависимость индекса а, определенного указанным способом, от размерности каркаса углеродных нанотрубок $D_{N}$, рассчитанной согласно
Сравнение значений фрактальной размерности структуры нанокомпозитов ПММА/МУНТ, определенных двумя методами

\begin{tabular}{c|c|c}
\hline$\varphi_{n}$ & $d_{f}$, уравнение $(4)$ & $d_{f}$, уравнение $(15)$ \\
\hline 0.0003 & 2.703 & 2.704 \\
0.0005 & 2.727 & 2.711 \\
0.0010 & 2.769 & 2.726 \\
0.0015 & 2.805 & 2.734 \\
0.0030 & 2.631 & 2.533 \\
0.0060 & 2.702 & 2.590
\end{tabular}

уравнению (3). Как следует из данных рис. 2, наблюдается линейное снижение $a$ по мере роста $D_{N}$, предполагающее рост степени усиления $E_{n} / E_{m}$ нанокомпозитов при прочих равных условиях. Для проверки общности приведенной на рис. 2 корреляции $a\left(D_{N}\right)$ использованы данные работы [20] для нанокомпозитов полиамид-6/углеродные нанотрубки (ПА-6/УНТ), которые подтверждают предполагаемую общность, по крайней мере, для нанокомпозитов полимер/углеродные нанотрубки. Представленную на рис. 2 корреляцию $a\left(D_{N}\right)$ можно выразить следующим эмпирическим уравнением:

$$
a=1.6-\left(D_{f}-1\right)
$$

или

$$
a=2.6-D_{f} .
$$

Сочетание уравнений (11) и (13) позволяет получить формулу для определения степени усиления нанокомпозитов полимер/углеродные нанотрубки:

$$
\frac{E_{n}}{E_{m}}=1+11\left(\varphi_{n}\right)^{2.60-D_{f}} .
$$

Отметим, что уравнение (14) подтверждает постулат авторов [21], которые предположили, что свойства полимерных нанокомпозитов контролируются структурой нанонаполнителя, формируемой в полимерной матрице. Кроме того, авторы [10] показали взаимосвязь размерностей $d_{f}$ и $D_{N}$, определяемую следующим соотношением:

$$
d_{f}=1.86+0.38 D_{N}
$$

Сравнение величин фрактальной размерности $d_{f}$ структуры нанокомпозитов ПММА/МУНТ, рассчитанных согласно уравнениям (4) и (15), приведено в таблице. Как следует из данных таблицы, указанные методы расчета $d_{f}$ дали хорошее соответствие (их среднее расхождение составляет $\sim 2 \%$ ). Уравнение (15) предполагает, что формирование каркаса частиц (агрегатов частиц) нанонаполнителя приводит к росту $d_{f}$ и в интервале типичных значений $D_{N}=1-3$ величина $d_{f}$ варьирует в пределах 2.24-3.0. Отсутствие указанного каркаса $\left(D_{N}=0\right)$ может привести к формированию пористой полимерной матрицы нанокомпозита $\left(d_{f}<2\right)$ и резкому снижению его степени усиления. 


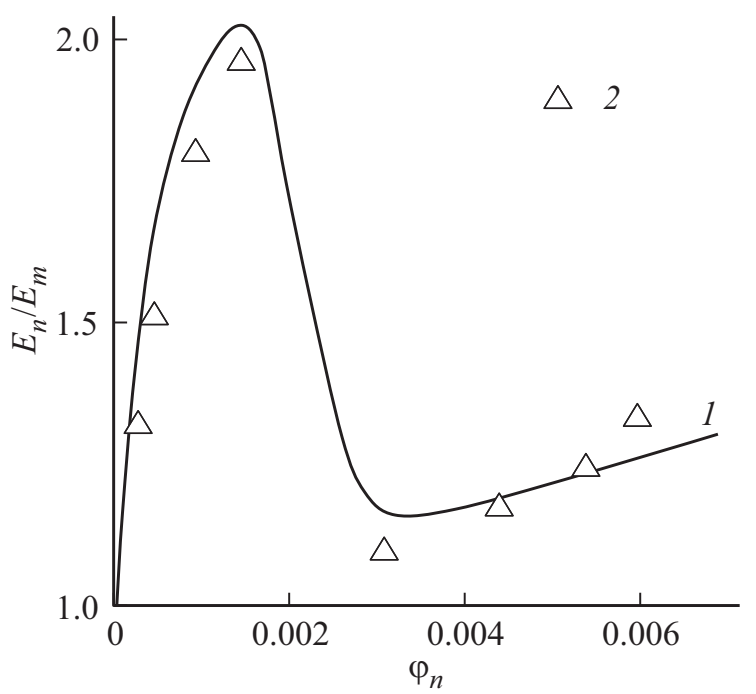

Рис. 3. Сравнение рассчитанной, согласно уравнению (14), (1) и полученной экспериментально (2) зависимостей степени усиления $E_{n} / E_{m}$ от объемного содержания нанонаполнителя $\varphi_{n}$ для нанокомпозитов ПММА/МУНТ.

На рис. 3 приведено сравнение рассчитанной согласно уравнению (14) и полученной экспериментально зависимостей $E_{n} / E_{m}\left(\varphi_{n}\right)$ для нанокомпозитов ПММА/МУНТ, которое показало хорошее соответствие предложенной модели и эксперимента (их среднее расхождение составляет $\sim 3 \%$ ).

И в заключение рассмотрим физический смысл размерности $D_{N}$, наиболее общим определением которой является следующее соотношение [10]:

$$
D_{N}=\frac{\ln N}{\ln \rho}
$$

где $N$ - число частиц размером $\rho$.

Из уравнения (16) следует, что при фиксированном содержании частиц увеличение $D_{N}$ определяет повышение $N$ при одновременном снижении/уменьшении степени агрегации нанонаполнителя $\rho$. Именно величина $\rho$ является наиболее важным фактором, определяющим свойства полимерных нанокомпозитов [15].

\section{Заключение}

Таким образом, результаты работы продемонстрировали, что структура углеродных нанотрубок в полимерной матрице, характеризуемая фрактальной размерностью их каркаса, контролирует свойства нанокомпозитов полимер/углеродные нанотрубки. Достижение порога перколяции нанонаполнителя приводит к существенным как качественным, так и количественным изменениям указанной размерности. Размерность каркаса углеродных нанотрубок определяет структурное состояние нанокомпозита как системы.

\section{Конфликт интересов}

Авторы заявляют, что у них нет конфликта интересов.

\section{Список литературы}

[1] Schaefer D.W., Justice R.S. // Macromolecules. 2007. Vol. 40. N 24. P. $8501-8517$. DOI: $10.1021 / \mathrm{ma} 070356 \mathrm{w}$

[2] Kim H., Abdala A.A., Macosko C.W. // Macromolecules. 2010. Vol. 43. N 16. P. 6515-6530. DOI: $10.1021 / \mathrm{ma} 100572 \mathrm{e}$

[3] Sun X., Sun H., Li H., Peng H. // Adv. Mater. 2013. Vol. 25. N 37. P. 5153-5177. DOI: 10.1002/adma.201301926

[4] Omidi M., Hossein Rokni D.T., Milani A.S., Seethaler R.J., Arasteh R. // Carbon. 2010. Vol. 48. N 11. P. 3218-3228. DOI: 10.1016/j.carbon.2010.05.007

[5] Blond D., Barron V., Ruether M., Ryan K.P., Nicolosi V., Blau W.J., Coleman J.N. // Adv. Funct. Mater. 2006. Vol. 16. N 15. P. 1608-1614. DOI: $10.1002 /$ adfm.200500855

[6] Khan U., May P., O'Neill A., Bell A.P., Boussac E., Martin A., Semple J., Coleman J.N. // Nanoscale. 2013. Vol. 5. N 3. P. 581-587. DOI: $10.1039 / \mathrm{c} 2 \mathrm{nr} 33049 \mathrm{k}$

[7] Микитаев А.К., Козлов Г.В. // ФТТ. 2015. Т. 57. Вып. 5. C. 961-964. [Mikitaev A.K., Kozlov G.V. // Physics Solid State. 2015. Vol. 57. N 5. P. 974-977. DOI: $10.1134 / \mathrm{S} 1063783415050224]$

[8] Vermant J., Ceccia S., Dolgovskij M.K., Maffettone P.L., Macosko C.W. // J. Rheology. 2007. Vol. 51. N 3. P. 429 450. DOI: $10.1122 / 1.2516399$

[9] Козлов Г.В.Фрактальная механика полимеров. М.: Изд-во Спутник +, 2016. 356 c.

[10] Козлов Г.В., Долбин И.В. // Известия вузов. Физика. 2018. T. 61. Вып. 6. C. 151-154. [Kozlov G.V., Dolbin I.V. // Russ. Phys. J. 2018. Vol. 61. N 5. P. 974-978.

DOI: $10.1007 / \mathrm{s} 11182-018-1485-4]$

[11] Rammal R., Toulouse G. // J. Phys. Lett. 1983. Vol. 44. N 1. P. L13-L22. DOI: 10.1051/jphyslet:0198300440101300

[12] Shady E., Gowayed Y. // Composites Sci. Tech. 2010. Vol. 70. N 10. P. 1476-1481. DOI: 10.1016/j.compscitech.2010.04.027

[13] Martone A., Faiella G., Antonucci V., Giordano M., Zarrelli M. // Composites Sci. Tech. 2011. Vol. 71. N 8. P. 1117-1123. DOI: 10.1016/j.compscitech.2011.04.002

[14] Yanovsky Yu.G., Kozlov G.V., Zhirikova Z.M., Aloev V.Z., Karnet Yu.N. // Intern. J. Nanomechan. Sci. Technol. 2012. Vol. 3. N 2. P. $99-124$.

DOI: 10.1615/NanomechanicsSciTechnolIntJ.v3.i2.10

[15] Микитаев А.К., Козлов Г.В., Заиков Г.Е. Полимерные нанокомпозиты: многообразие структурных форм и приложений. М.: Наука, 2009. 278 с.

[16] Hentschel H.G.E., Deutch J.M. // Phys. Rev. A. 1984. Vol. 29. N 3. P. 1609-1611. DOI: 10.1103/PhysRevA.29.1609

[17] Микитаев А.К., Козлов Г.В. // ДАН. 2015. Т. 462. Вып. 1. C. 41-44. [Mikitaev A.K., Kozlov G.V. // Doklady Physics. 2015. Vol. 60. N 5. P. 203-205.

DOI: $10.1134 / \mathrm{S} 102833581505002 \mathrm{X}$

[18] Bridge B. // J. Mater. Sci. Lett. 1989. Vol. 8. N 2. P. 102-103. DOI: $10.1007 / \mathrm{BF} 00720265$

[19] Микитаев А.К., Козлов Г.В. // ФТТ. 2017. Т. 59. Вып. 7. C. 1418-1421. [Mikitaev A.K., Kozlov G.V. // Phys. Solid State. 2017. Vol. 59. N 7. P. $1446-1449$. DOI: $10.1134 / \mathrm{S} 1063783417070149]$

[20] Gao J., Itkis M.E., Yu A., Bekyarova E., Zhao B., Haddon R.C. // J. Amer. Chem. Soc. 2005. Vol. 127. N 11. P. 3847-3854. DOI: $10.1021 /$ ja0446193

[21] Schaefer D.W., Zhao J., Dowty H., Alexander M., Orler E.B. // Soft. Matter. 2008. Vol. 4. N 10. P. 2071-2079. DOI: $10.1039 / \mathrm{b} 805314 \mathrm{f}$ 